\title{
SYMMETRY RECOGNITION APPLIED TO TWO-DIMENSIONAL NMR DATA
}

\author{
by
JEFFREY C. HOCH $^{1) a)}$, SHEN HENGYI ${ }^{2)}$, MOGENS KJ $\AA^{2)}$, SVEND LUDVIGSEN ${ }^{2)}$
and \\ FLEMMING M. POULSEN ${ }^{2) a)}$
}

1) Rowland Institute for Science, 100 Cambridge Parkway, Cambridge, Massachusetts 02142.

\author{
2) Department of Chemistry, Carlsberg Laboratory, \\ Gamle Carlsberg Vej 10, DK-2500 Copenhagen Valby \\ a) To whom correspondence should be addressed
}

Keywords: Symmetry recognition, pattern recognition, two-dimensional NMR, automated analysis, group theory

\begin{abstract}
A procedure which automatically locates features in two dimensional NMR spectra based on their symmetry properties is described. The procedure makes use of projection operators from group theory. Results of the procedure applied to COSY data for the peptide hormone gonadoliberin and the barley serine protease inhibitor 2 (BSI 2) are presented.
\end{abstract}

\section{INTRODUCTION}

Interest in automated analysis of two-dimensional NMR spectra $(1,5,6,7)$ is motivated by the high information content and complexity of 2D-NMR spectra, which conspire to make the manual analysis of the spectra of large moleculès, such as proteins, a tedious task. Further motivation is provided by the redundancy inherent in 2D-NMR spectra. The positions of the four cross peaks in a shift correlation experiment arising from an $\mathrm{AX}$ spin system contain only three unique pieces of information: two chemical shifts and a coupling constant. It is therefore desirable to develop methods for automating the analysis of two-dimensional
NMR spectra, not only to eliminate a tedious task, but also to make maximum use of redundant information present in the spectra in order to obtain reliable spectral parameters.

Recent progress toward automated spectrum analysis has been reported using a pattern recognition technique (6). In this method a template is passed over the spectrum, and a reduced spectrum is constructed which is a measure of the degree of match between the template and the input data. This procedure is repeated using a series of templates of different sizes corresponding to the range of possible coupling constants. The result is a four-dimensional surface $\mathrm{g}\left(\omega_{1}, \omega_{2}, \mathrm{~J}\right)$. Local maxima occur on this surface

Abbreviations: BSI 2 = barley serine protease inhibitor 2 ; COSY $=$ two-dimensional ${ }^{1} \mathrm{H}$ homonuclear correlation spectroscopy; NMR = nuclear magnetic resonance; NOESY = two-dimensional nuclear Overhauser spectroscopy. 
at positions corresponding to a multiplet in the spectrum. The position of a local maximum gives the chemical shift in both frequency dimensions of the center of the multiplet and the magnitude of the coupling constant.

A different approach to the identification of the significant features in a 2D-NMR spectrum is that of symmetry recognition, rather than pattern recognition. By symmetry recognition we mean the identification of objects which are invariant under the application of a particular group of operations. Since symmetry is scale invariant, symmetry recognition, in principle, identifies features exhibiting specified symmetry elements independent of the magnitute of coupling constants or linewidths. In the present communication we illustrate a procedure for performing symmetry recognition using group theory projection operators.

\section{MATERIALS AND METHODS}

Proton NMR spectra were recorded on a Bruker AM 500 NMR spectrometer equipped with an Aspect 3000 computer. The two-dimensional NMR spectra were recorded in the phase sensitive mode using the method proposed by STATES et al. (8) for NOESY experiments. Typically, the two-dimensional NMR spectra were accumulated with 512 complex $t_{1}$ values and 2048 complex $t_{2}$ values using quadrature detection in both dimensions. The data were processed using zerofilling to 2048 complex points in the $t_{1}$ dimension and 8192 complex points in the $t_{2}$ dimension. All data processing was performed on a VAX 11/750 using the Carlsberg Laboratory NMR software which use some routines from the Rowland NMR Toolkit (3).

Gonadoliberin was a gift from Carlbiotech Ltd. (Copenhagen, Denmark) and the barley serine protease inhibitor was purified as described by JONASSEN et al. (4).

\section{THEORY}

A two-dimensional NMR spectrum may be considered a three-dimensional surface $f\left(\omega_{1}, \omega_{2}\right)$, and consequently the symmetry properties of entities within the spectrum may be classified as belonging to one of the familiar point groups (2).
Three levels of symmetry may be distinguished in 2D-NMR spectra: symmetry of the spectrum as a whole, symmetry of an individual resonance, and symmetry of groups of resonances (multiplets). Reflection symmetry about the diagonal is a familiar symmetry of the overall spectrum. Individual resonances exhibit local symmetry. For example, a pure absorption lorentzian peak having the same linewidths in both dimensions has four reflection planes, two $\frac{\pi}{2}$ rotation axes, a $\pi$ rotation axis, and the identity operation as symmetry elements, classifying it as belonging to the point group $\mathrm{C}_{4 \mathrm{v}}$. A peak which is dispersive in both dimensions belongs to the group $\mathrm{D}_{2 \mathrm{~d}}$. Multiplets likewise exhibit local symmetry. Examples of the three levels of symmetry present in 2D-NMR spectra are shown in Figure 1.

The concept from group theory that provides a means of performing symmetry recognition is the projection operator. The projection operator corresponding to the $\mathrm{j}^{\text {th }}$ irreducible representation of a group is given by (2):

$$
P_{j} \equiv\left(l_{j} / n\right) \sum_{R} \chi^{j}(R) * r
$$

where $\mathrm{l}_{\mathrm{j}}$ is the dimension of the $\mathrm{j}^{\text {th }}$ irreducible representation, $\mathbf{n}$ is the order of the group, $\mathbf{R}$ represents the symmetry operations of the group, $\chi^{\mathrm{j}}(\mathrm{R})$ is the character of the operation $\mathrm{R}$ in the $\mathrm{j}^{\text {th }}$ irreducible representation, and $\mathrm{r}$ is the operator (applied to functions). The effect of a projection operator $P_{j}$ on an arbitrary function is to "project out" that portion of the function which spans the irreducible representation $\Gamma^{\mathrm{j}}$ of the group, eliminating components lacking the appropriate symmetry.

Construction of the projection operator for the $A^{\prime}$ irreducible representation of the point group $\mathrm{C}_{\mathrm{s}}$ serves to illustrate the operations involved, and demonstrates that projection operators are already familiar to NMR spectroscopists. Group $C_{s}$ has only two symmetry operators: $E$, the identity, and $\sigma_{\mathrm{d}}$, reflection through a mirror plane which passes through the line $\omega_{1}=\omega_{2}$ perpendicular to the plane $f\left(\omega_{1}, \omega_{2}\right)$ $=0$. The projection operator for the totally symmetric irreducible representation is thus 
J. C. Hoch et al.: Symmetry recognition in 2D-NMR spectroscopy

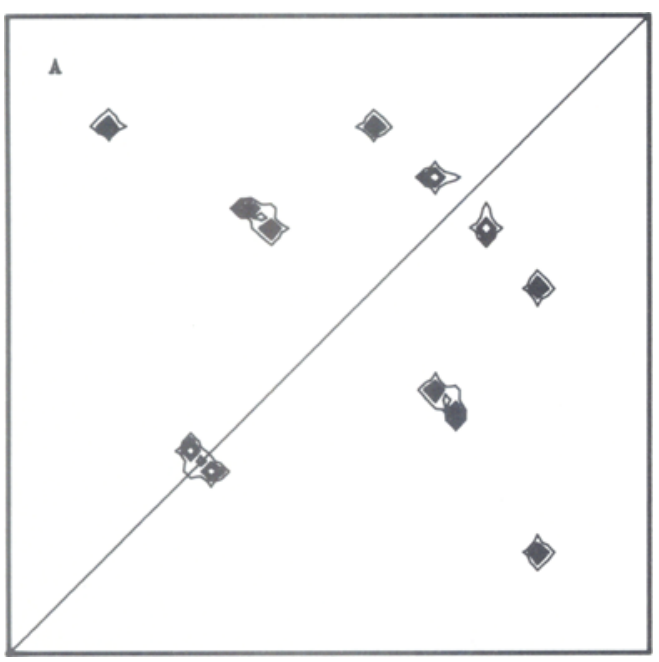

$$
P=\frac{1}{2}\left(E+\sigma_{d}\right)
$$

and the result of applying this operator to an arbitrary point $\mathrm{f}\left(\omega_{1}, \omega_{2}\right)$ is

$$
\frac{1}{2}\left[f\left(\omega_{1}, \omega_{2}\right)+f\left(\omega_{2}, \omega_{1}\right)\right]
$$

which, for this point group, is simply the average of the symmetry related points, a commonly used method for symmetrizing spectra about the diagonal.

Symmetrizing spectra about the diagonal requires only one projection operator, since the symmetry is global. The local symmetry of resonances or multiplets cannot be exploited in the same way, however. Implicit in the operators

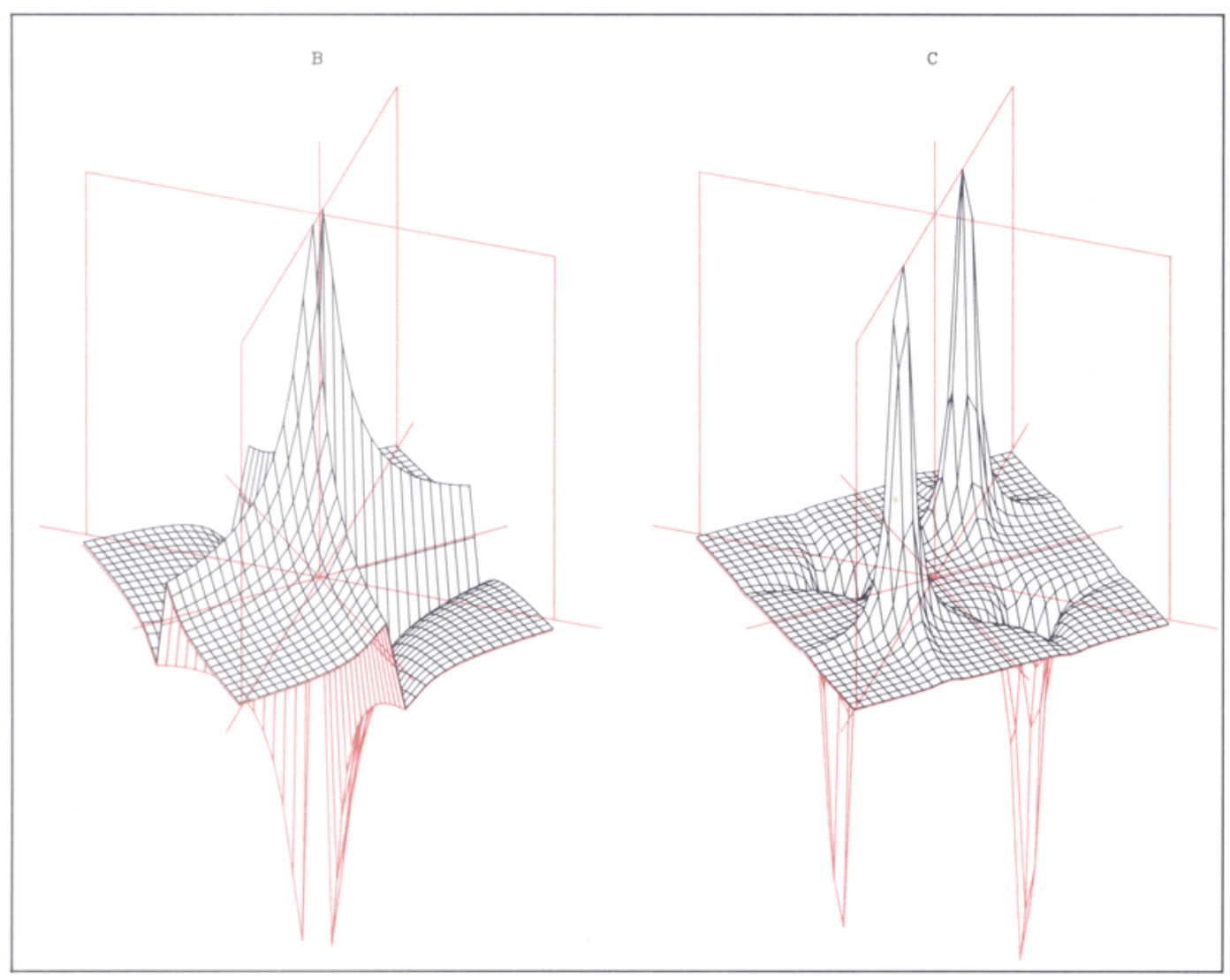

Figure 1. Examples of symmetry in 2D-NMR spectra. A) Diagonal symmetry (global): contour diagram of two-dimensional NMR spectrum with diagonal symmetry. B) Resonance symmetry (local): the example shown is a cross peak dispersive in both dimensions. C) Multiplet symmetry (local): an antiphase cross peak of an AX spin system in a phase sensitive COSY spectrum. In (B) and (C) the symmetry operations for the $D_{2 d}$ point group are indicated. 


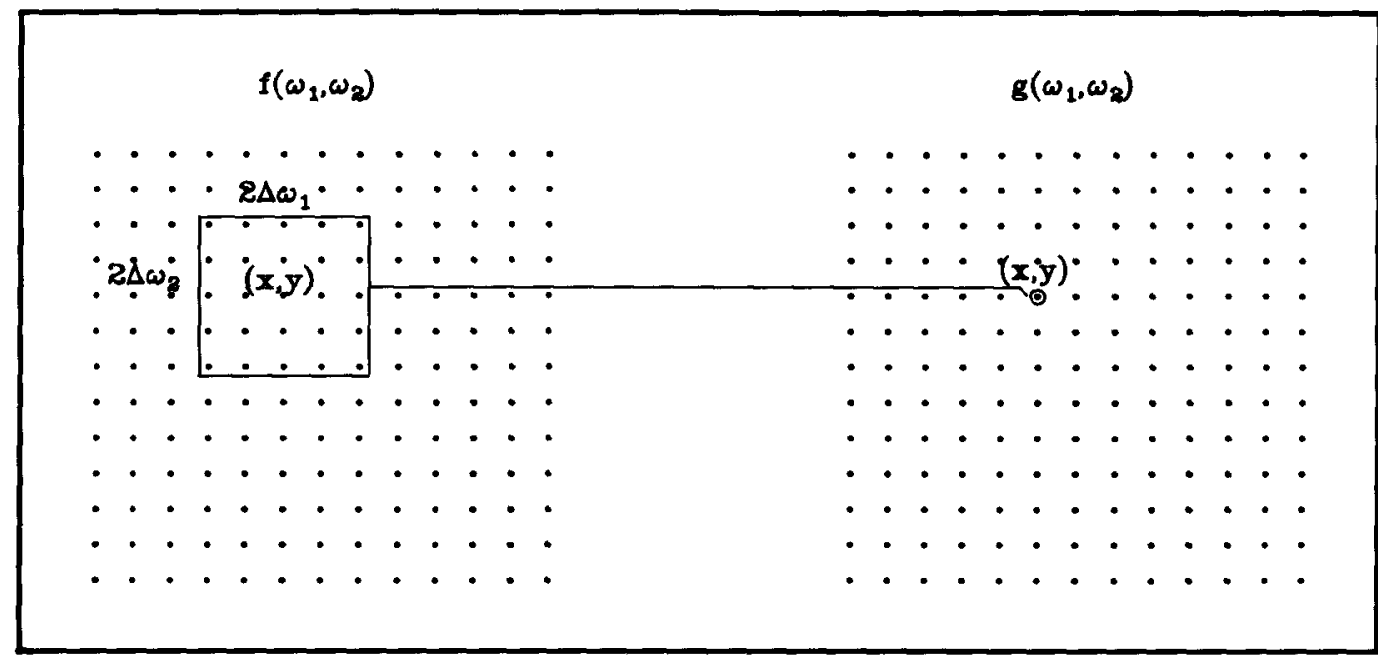

Figure 2. Points utilized in the computation of $g\left(\omega_{1}, \omega_{2}\right)$ for performing symmetry recognition.

$r$ in equation ( 1 ) is the center, or origin, about which the operations apply. Consequently a projection operator centered on a single origin will not serve to project out all features having the same local symmetry, since each feature has its own center of symmetry. A moving projection operator will project all features exhibiting local symmetry corresponding to that of the projection operator, if the projection operator is applied about every possible center of symmetry. The output of the projection operator will exhibit a local maximum whenever the origin of the projection operator coincides with the center of a feature exhibiting the appropriate symmetry. The fall-off of the output of the projection operator as its origin is displaced from a local center of symmetry provides a means of automatically identifying local centers of symmetry.

A general scheme for using projection operators to locate spectral features exhibiting a desired local symmetry consists of the following steps. First the local symmetry elements to be sought are determined, or equivalently the point group corresponding to the local symmetry. Next the projection operator for one of the irreducible representations of the point group is constructed for an arbitrary point $\left(\omega_{1}, \omega_{2}\right)$ of the two-dimensional grid on which the spectrum is defined. By choosing the totally symmetric rep- resentation, that is the one for which the character $\chi(r)$ is 1 for all symmetry operations $\mathrm{r}$, the construction of the projection operator is simplified, and furthermore assures that the projection operator reflects the influence of all of the symmetry operations of the group. At each possible origin, a measure $g\left(\omega_{1}, \omega_{2}\right)$ of the output of the projection operator centered on that point is computed. This measure is computed from the values fo the projection operator applied to an array of points $\left(\omega_{1} \pm \Delta \omega_{1}, \omega_{2} \pm \Delta \omega_{2}\right)$ centered about the local origin; the array (illustrated in Figure 2) must be large enough to encompass the spectral feature being sought (or a significant fraction of it). Finally, the local centers of symmetry in the spectrum are identified by locating the local maxima of the output function $\mathrm{g}\left(\omega_{1}, \omega_{2}\right)$.

The measure $g\left(\omega_{1}, \omega_{2}\right)$ can be computed in a number of ways. One simple example is to compute the sum of the values obtained from applying the projection operator to each of the points of the array centered on $\left(\omega_{1}, \omega_{2}\right)$,

$$
g\left(\omega_{1}, \omega_{2}\right)=\sum_{\substack{\omega_{1} \leq x \leq \omega_{1}+\Delta \omega_{1} \\ \omega_{2} \leq y \leq \mathrm{W}}} P[f(x, y)]
$$

where $W$ is equal to $\omega_{2}+\Delta \omega_{2}$ for the point groups 


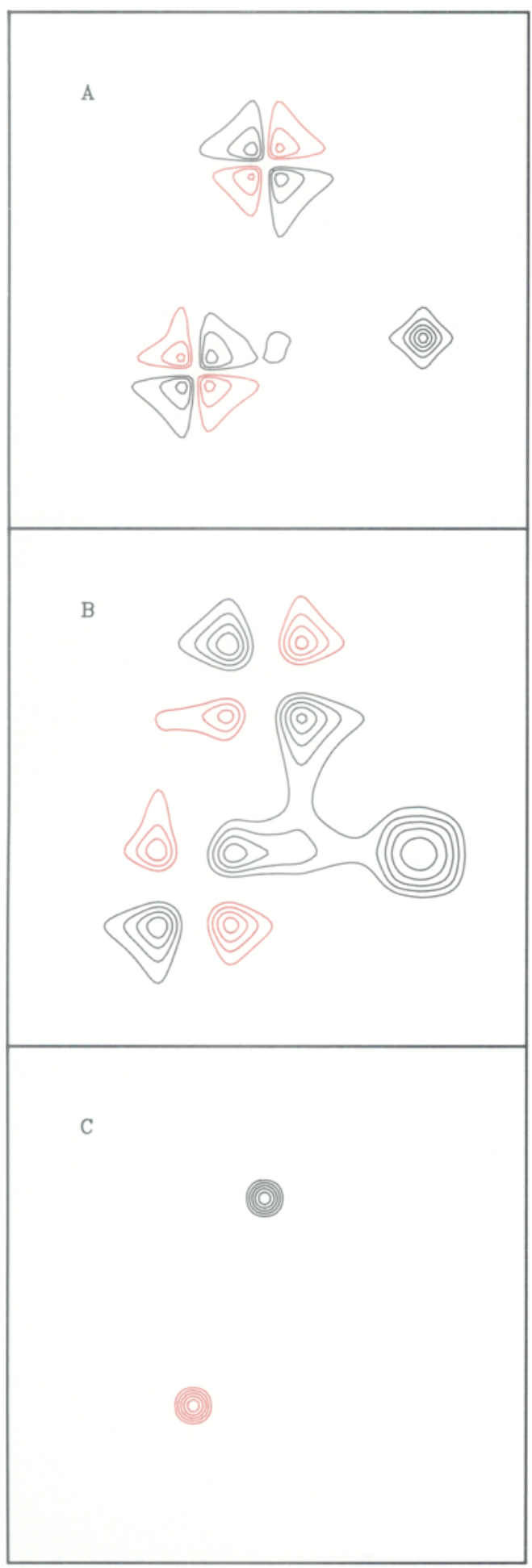

Figure 3. Projection operators applied to a synthetic spectrum containing a pure absorption peak $\left(C_{4 v}\right)$ and two dispersive peaks $\left(D_{2 d}\right)$, using the measure of equation (4), A) contour plot for the starting spectrum, B) contour plot for $g\left(\omega_{1}, \omega_{2}\right)$ obtaining with a $C_{4 v}$ projection operator, $C)$ contour plot for $g\left(\omega_{1}, \omega_{2}\right)$ obtained with a $D_{2 d}$ projection operator.

$\mathrm{C}_{2 \mathrm{v}}$ or $\mathrm{D}_{2}$, and is equal to $\mathrm{x}$ for the point groups $\mathrm{C}_{4 \mathrm{v}}$ and $\mathrm{D}_{2 \mathrm{~d}}$. These limits on the explicit summation in equation (4) assure that unique values of the magnitude of $P[f(x, y)]$ are computed only once, and furthermore assures that the sum does not vanish for point groups having symmetry elements which produce sign reversal $\left(D_{2 d}\right.$ or $D_{2}$ ). Figure 3 illustrates the results of this method for computing $g\left(\omega_{1}, \omega_{2}\right)$ when applied to a synthetic spectrum containing a pure absorption peak and two dispersive peaks having opposite sign. Equivalent linewidths were used for both dimensions in generating the synthetic spectrum, so the absorption peak spans the point group $C_{4 v}$ and the dispersive peaks span $D_{2 d}$. Figure $3 \mathrm{~A}$ illustrates the starting spectrum. Contour diagrams of the corresponding measure $\mathrm{g}\left(\omega_{1}, \omega_{2}\right)$ are shown in Figures $3 \mathrm{~B}$ and $3 \mathrm{C}$ for the projection operators of the totally symmetric irreducible representations of $C_{4 v}$ and $D_{2 d}$, respectively. Note that the lobes of the dispersive peaks exhibit some $\mathrm{C}_{4 \mathrm{v}}$ character, giving rise to peaks in the reduced spectrum located about the center of the dispersive peak. The peak which appears at the center of the pure absorption peak, while larger than these peaks, is quite broad. Consequently, the maximum is poorly defined and overlap may prove severe for crowded spectra. The reduced spectrum obtained using the $D_{2 d}$ projection operator (Figure $3 C$ ) is far more selective, and the peak identifying the location of the dispersive peak in the starting spectrum is more sharply defined.

The measure $\mathrm{g}\left(\omega_{1}, \omega_{2}\right)$ can be made more selective by including a penalty term which diminishes the contribution made by a point to the reduced spectrum if the range of values of symmetry related points is large. BOLTON has used such a penalty term in the application of symmetry filters in one dimension (1). A simple extension of Bolton's penalty term to the present 


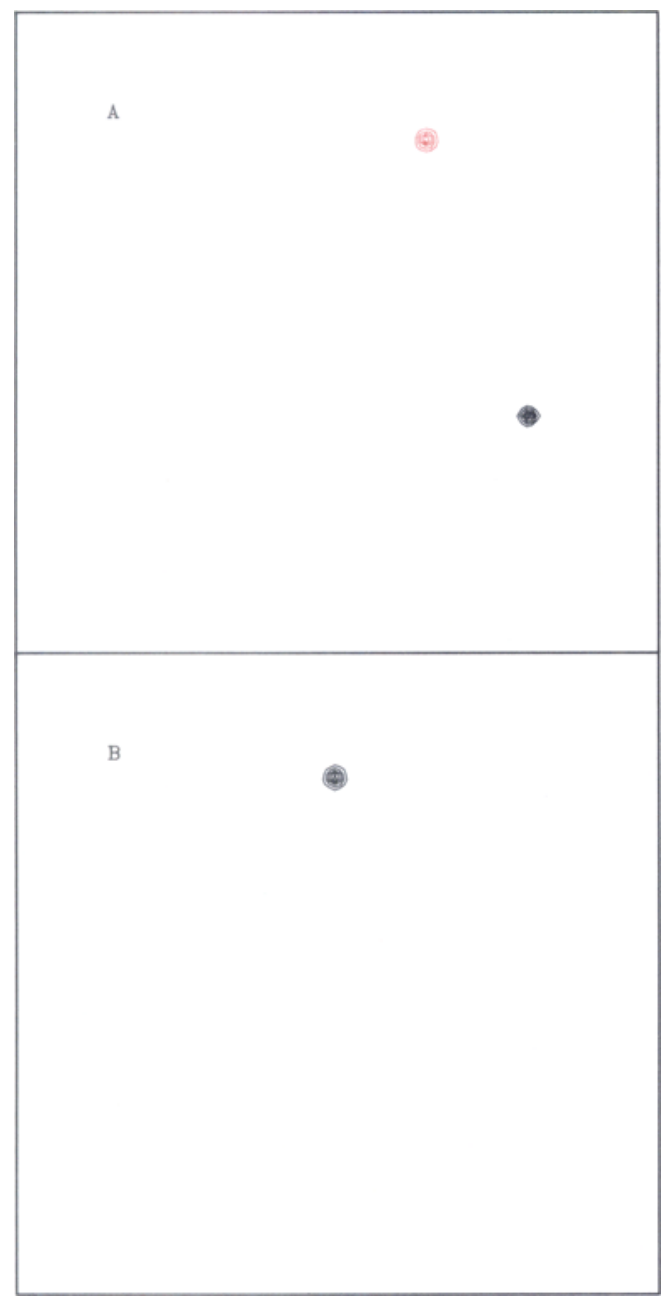

Figure 4. Projection operators applied to the spectrum of Figure $3 \mathrm{~A}$ using the measure of equation (5), $\mathrm{A}$ ) contour plot for $g\left(\omega_{1}, \omega_{2}\right)$ obtained with $\mathrm{a}_{4 \mathrm{v}}$ projection operator, B) contour plot for $g\left(\omega_{1}, \omega_{2}\right)$ obtained with a $\mathrm{D}_{2 \mathrm{~d}}$ projection operator.

case (where there are more than two symmetry related values) is the measure

$$
g\left(\omega_{1}, \omega_{2}\right)=\sum_{\substack{\omega_{1} \leq x \leq \omega_{1}+\Delta \omega_{1} \\ \omega_{2} \leq y \leq \mathrm{W}}} P[f(x, y)]\left(1-Q^{2}(x, y)\right)
$$

where $Q(x, y)$ is given by

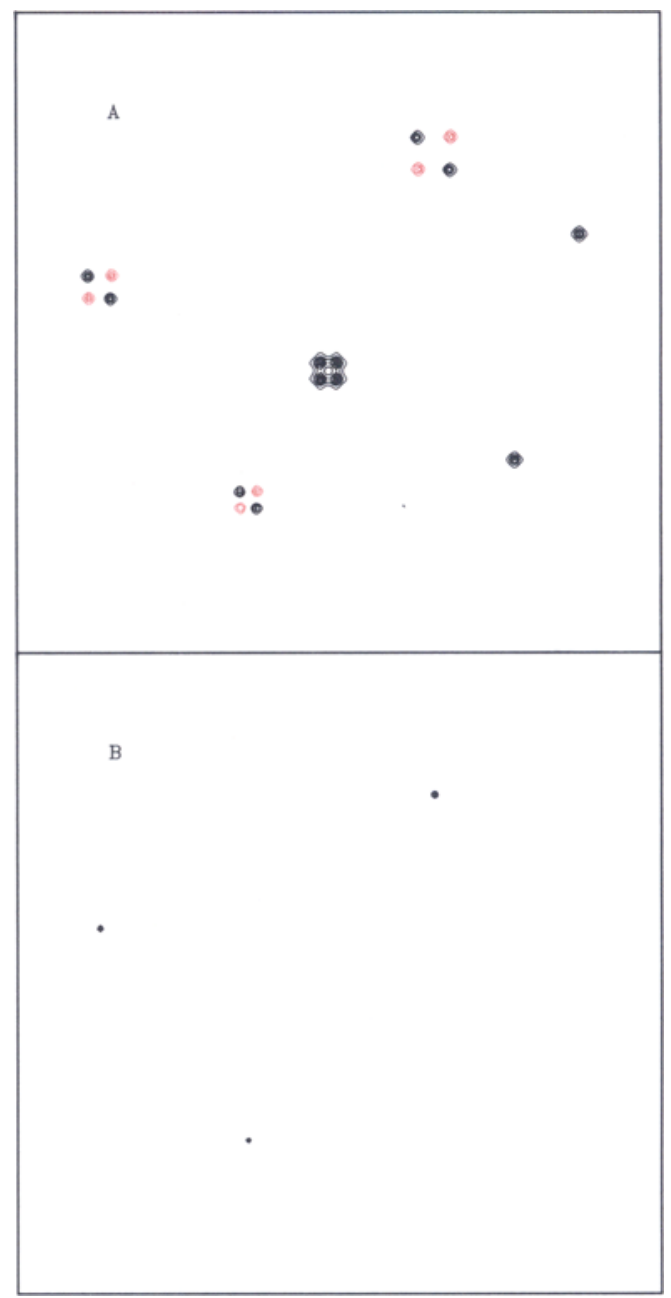

Figure 5. Projection operators applied to a synthetic spectrum containing multiplets with $D_{2 d}$ and $C_{4 v}$ symmetry, using the measure of equation (5), A) contour plot for the starting spectrum, B) contour plot for $g\left(\omega_{1}, \omega_{2}\right)$ obtained with a $D_{2 d}$ projection operator.

$$
\frac{\left.M A X\right|_{R}|f(x, y)|-\left.M I N\right|_{R}|f(x, y)|}{\left.M A X\right|_{R}|f(x, y)|+\left.M I N\right|_{R}|f(x, y)|}
$$

and R (the set of symmetry operations) denotes that the maximum and minimum values of $f(x, y)$ are selected from the symmetry related points. Contour plots of the reduced spectra obtained by applying the measure given by equation (5) to the spectrum of Figure $3 \mathrm{~A}$ are 
Figure 6. A portion of the $500 \mathrm{MHz}$ phase-sensitive COSY spectrum of gonadoliberin in $10 \% \mathrm{D}_{2} \mathrm{O}$ at $310 \mathrm{~K}$, pH 4.2. A data matrix of $4096 t_{2}$ points $512 t_{1}$ values was zerofilled to $4 \mathrm{~K}$ by $4 \mathrm{~K}$ complex points, with a final digital resolution of $1.82 \mathrm{~Hz}$ in both dimensions. In (A) contour levels are drawn at $1,2,3,5$, with negative contours drawn in red. (B) depicts the regions of interest determined using the list procedure described in the text. (C) illustrates the reduced spectrum computed for the regions outlined in (B) using equation (5) with a $D_{2 d}$ projection operator.

shown in Figures $4 A$ and $4 B$, using $C_{4 v}$ and $D_{2 d}$ projection operators, respectively. The results are far more selective for the desired symmetry and the features are more sharply defined than those obtained using the measure given by equation (4). Other measures $g\left(\omega_{1}, \omega_{2}\right)$ can be conceived which have similar properties. For example, an alternative to the penalty term $\left(1-Q^{2}(x, y)\right)$ is to use the probability, based on an f-test, that the variance computed for a set of symmetry related values of $f\left(\omega_{1}, \omega_{2}\right)$ exceeds the noise variance.

Figure 5 illustrates the use of symmetry recognition to identify antiphase multiplets. The starting (synthetic) spectrum (Figure 5A) contains three antiphase multiplets with 5, 7.5, and $10 \mathrm{~Hz}$ coupling constants. The contour plot of the reduced spectrum (Figure 5B), computed using the measure of equation (5) with a $D_{2 d}$ projection operator, clearly identifies the positions of the antiphase multiplets.

\section{RESULTS AND DISCUSSION}

\subsection{Practical symmetry recognition}

While, : in principle, the formal symmetry recognition procedure just described can be applied to large two dimensional data sets, in practice the computation of the reduced spectrum $g\left(\omega_{1}, \omega_{2}\right)$ according to equations (4) or (5) for each point of a large 2D data set is very time consuming. Fortunately, when viewed as images, 2D-NMR spectra are sparse. A practical algorithm for applying symmetry recognition to large 2D spectra would avoid computing $g\left(\omega_{1}, \omega_{2}\right)$ for empty regions of the spectrum. In this section we describe two simple procedures

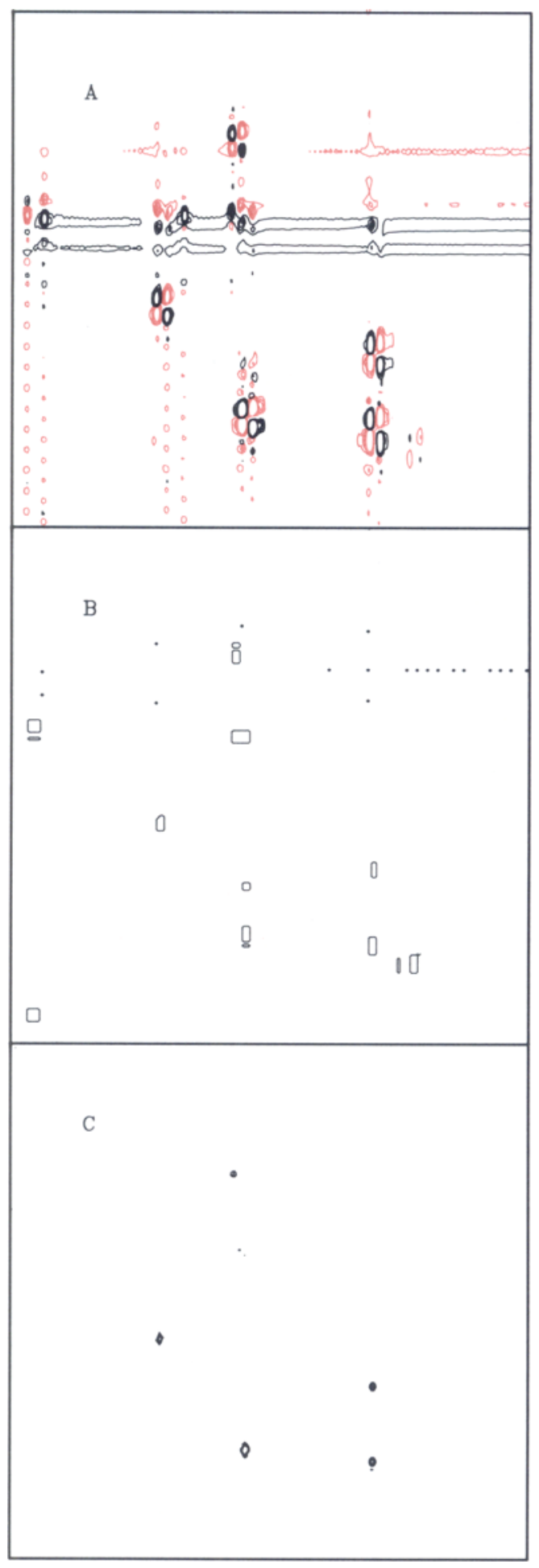




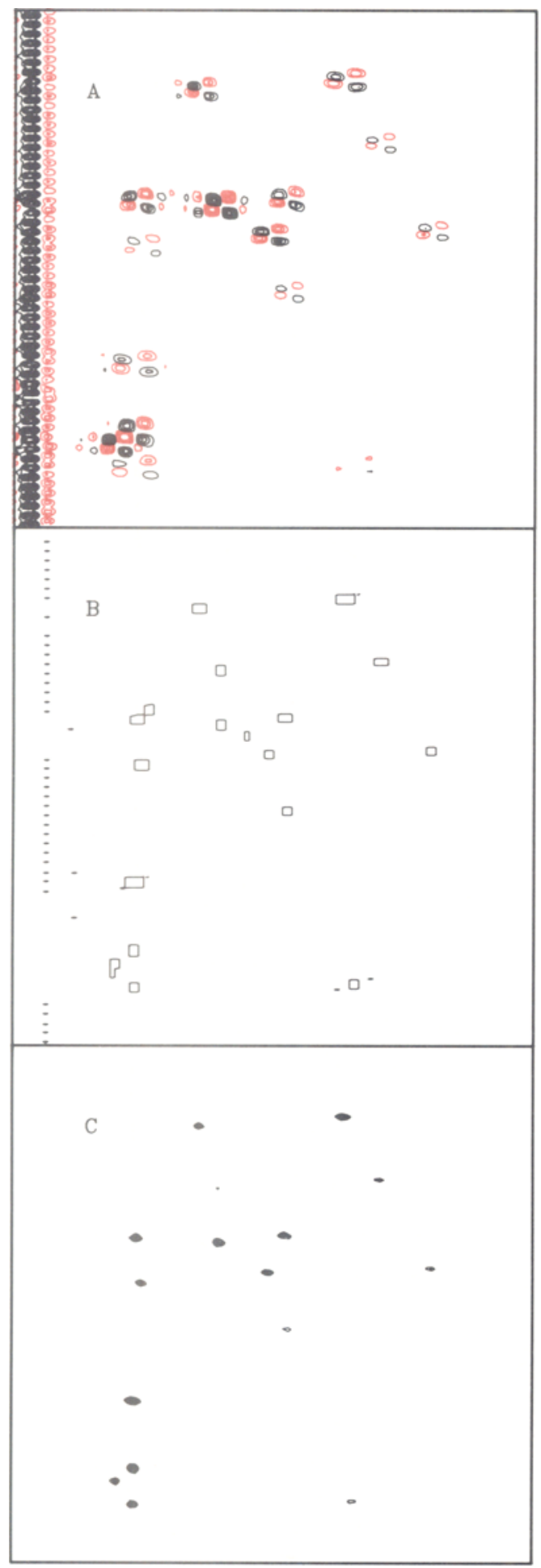

Figure 7. A portion of the $500 \mathrm{MHz}$ phase-sensitive COSY spectrum of BSI 2 obtained at $315 \mathrm{~K}, \mathrm{pH} 4.2$. A data matrix of $2048 t_{2}$ points at $426 t_{1}$ values was zerofilled to $8 \mathrm{~K}$ by $2 \mathrm{~K}$ complex points, with final digital resolution of $3.65 \mathrm{~Hz}$ in $\omega_{1}$ and $0.91 \mathrm{HZ}$ in $\omega_{2}$. In (A) contour levels are drawn at 1, 2, 3,5 with negative levels drawn in red. (B) depicts the regions of interest determined using the list procedure with a peak threshold of 0.7 and a distance cutoff of $14.6 \mathrm{~Hz}$. (C) shows the reduced spectrum computed for the regions outlined in (B) using equation (5) with a $\mathrm{D}_{2}$ projection operator.

for identifying "regions of interest", suitable for locating antiphase multiplets in COSY spectra, for which the reduced spectrum is subsequently computed.

The characteristic sign alternation of antiphase multiplets affords an opportunity for a simple definition of regions of interest as consisting of points for which peaks having the appropriate sign are located in each of the four quadrants of the spectrum relative to that point and within a distance just larger than half the largest $\mathrm{J}$ coupling in the spectrum. A hierarchical procedure for applying this test consists of the following steps. First, the points (both positive and negative) in the spectrum larger than a magnitude threshold are identified. Next, for each point of the spectrum, a list is constructed of the sign and direction (from that point) of each neighboring peak (within $\pm J_{\max } / 2$ in both dimensions). If a point is not within $\pm \mathrm{J}_{\max } / 2$ in both dimensions of a peak, then the corresponding list is empty. A list can contain at most eight entries, if there are both positive and negative peaks in all four quadrants of the spectrum relative to that point. Finally, the regions of interest for which the reduced spectrum is computed are identified as those points which have lists indicating a positive peak in the northeast and southwest quadrants (upper right and lower left), and a negative peak in the northwest and southeast quadrants (upper left and lower right). Figure 6 illustrates the application of this method to phase-sensitive COSY data for gonadoliberin. The reduced spectrum shown in Figure $6 \mathrm{C}$ was computed for the regions outlined in Figure 6B using equation (5) with a $D_{2 d}$ 


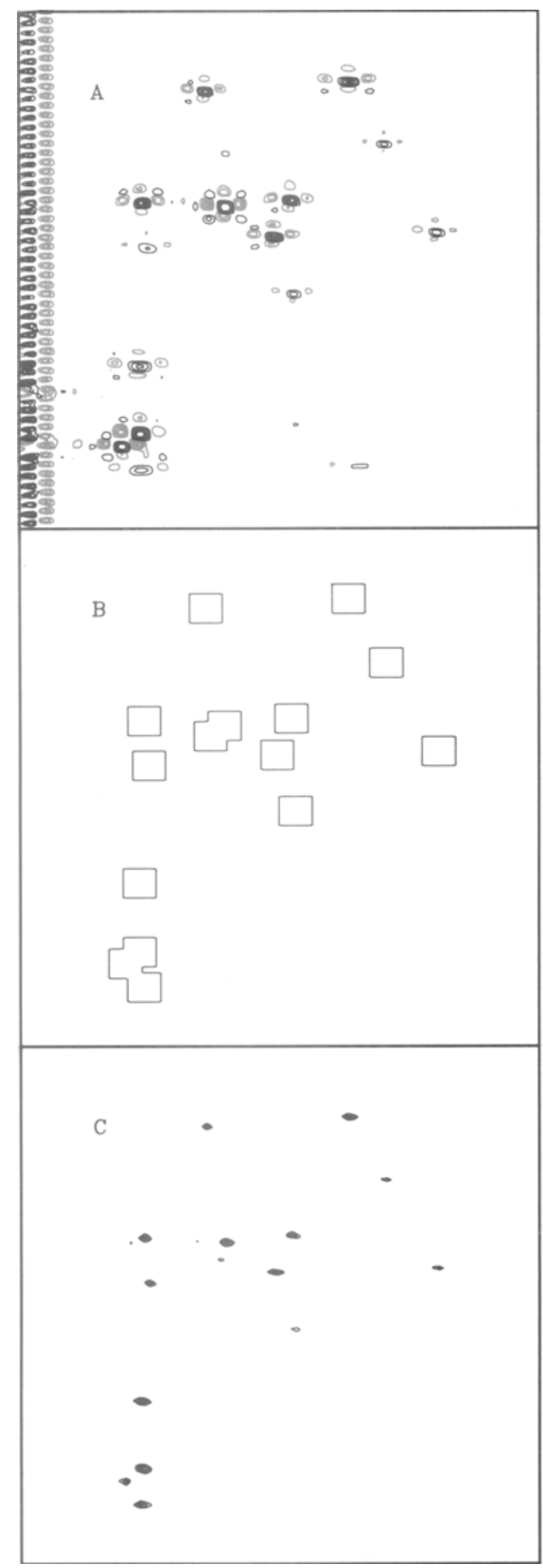

Figure 8. (A) The COSY data shown in Figure 7A to which a constant phase shift of $-90^{\circ}$ has been applied in both $\omega_{1}$ and $\omega_{2}$. Contour levels are drawn at $1,2,3$, 5 with negative levels drawn in red. (B) depicts regions of interest determined by all points within $11.0 \mathrm{~Hz}$ of a positive peak above a threshold of 2 .

projection operator. Note that application of a $D_{2 d}$ projection operator requires that the digital resolution be the same in $\omega_{1}$ and $\omega_{2}$. Despite the slightly different linewidths in $\omega_{1}$ and $\omega_{2}$, the antiphase multiplets exhibit sufficient symmetry to be located using a $D_{2 d}$ projection operator. Figure 7 illustrates similar results obtained for a portion of an $8 \mathrm{~K}$ by $2 \mathrm{~K}$ COSY spectrum of barley serine protease inhibitor 2 (BSI 2). The reduced spectrum shown in Figure $7 \mathrm{C}$ was obtained using equation (5) with a $\mathrm{D}_{2}$ projection operator.

Another opportunity for a simple test to identify regions which contain antiphase multiplets is provided by the relatively small values of the coupling constants typical of peptide and protein spectra. A consequence is that when the constant phase correction is decremented by $90^{\circ}$ in both dimensions, constructive interference occurs near the center of symmetry of the multiplet, giving rise to a peak larger than any of the others comprising the multiplet. Regions of interest can then be simply defined as points within a fixed distance of a positive peak above a chosen threshold. Regions of interest determined in this fashion for the data of Figure 7A are shown in Figure 8. The "skewed" spectrum obtained by decreasing the phases by $90^{\circ}$ from those which yield pure absorption peaks has the interesting property that the normally dispersive diagonal peaks (Figure 9) have pure absorption line shapes, permitting peaks which might otherwise be obscured by strong dispersive tails to be discerned more easily. It should also be noted that symmetry recognition can be performed on the skewed spectrum; antiphase multiplets now belong to the point group $\mathrm{C}_{4 \mathrm{v}}$ (or $\mathrm{C}_{2 \mathrm{v}}$ ). This provides a means of exploiting the redundancy inherent in the four quadrants of a hypercomplex spectrum.

The simple methods described here for determining regions of interest likely to contain 


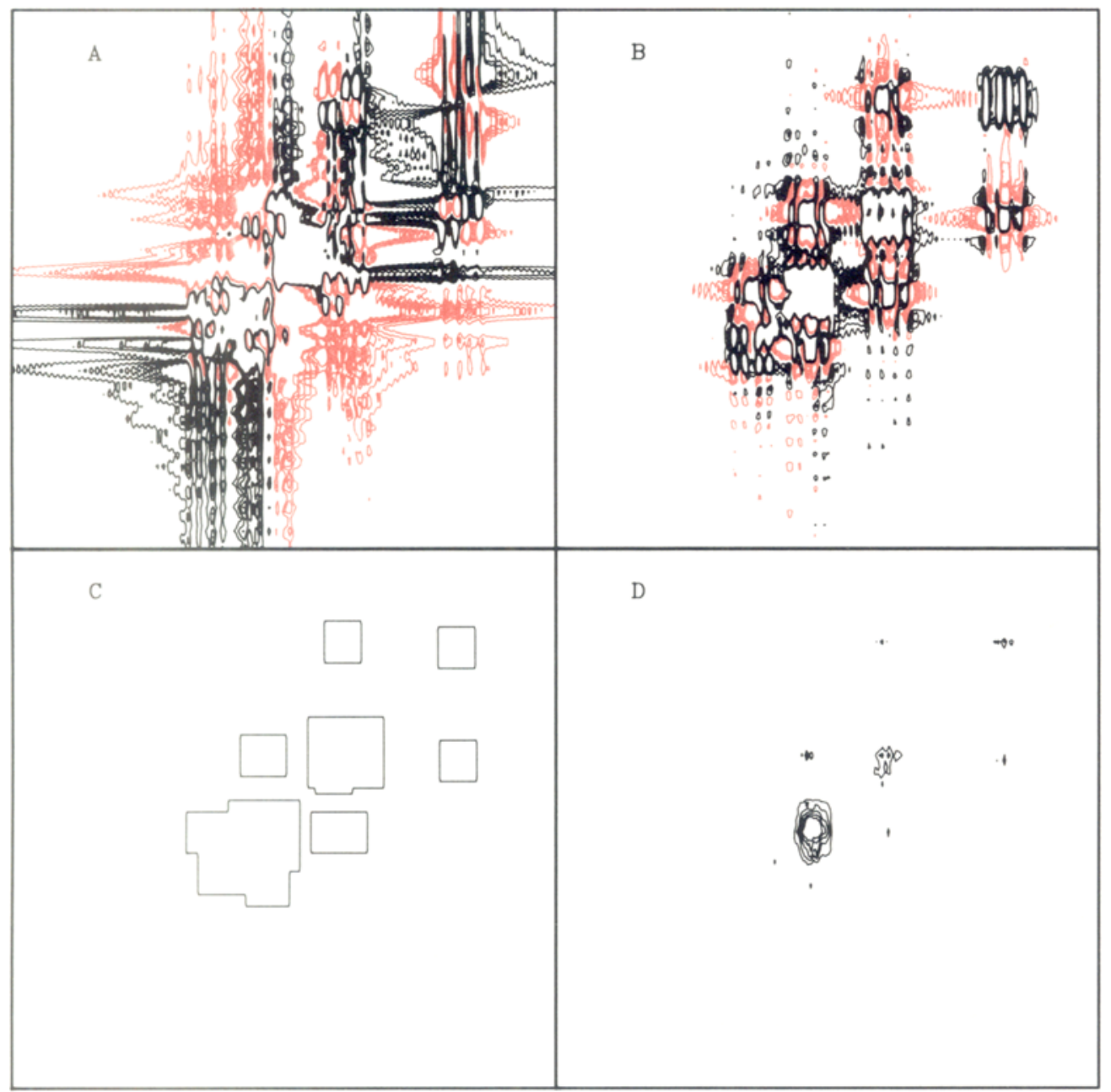

Figure 9. A portion of the $500 \mathrm{MHz}$ phase-sensitive COSY spectrum of gonadoliberin (310K, $\mathrm{pH}$ 4.2) illustrating points near the diagonal (A). The same dataset, that was used in Figure 6, however, zerofilled to $8 \mathrm{~K}$ by $2 \mathrm{~K}$ complex points. Contour levels are drawn at 5, 7,9 with negative levels drawn in red. (B) shows the "skewed" spectrum. (C) illustrates regions of the spectrum which are within $11.0 \mathrm{~Hz}$ of a positive peak larger than 20 . (D) illustrates the reduced spectrum obtained using equation (5) with a $D_{2}$ projection operator

antiphase multiplets are by no means exhaustive or without limitations. They are illustrative, however, of the kinds of simple and efficient computational algorithms which can be used in conjunction with moving projection operators to achieve practical symmetry recognition.

\subsection{Connections to previous work}

The use of moving projection operators is not unique to the procedure described here for performing symmetry recognition. Moving projection operators also form the basis for the symmetry filters described by BOLTON (1) and the pattern recognition procedure of PFÄNDLER et al. $(6,7)$, although they are not described as such and both treatments consider specific rather than general symmetry. One major difference between their use in those methods and the present method is that fewer symmetry elements are exploited because the projection 


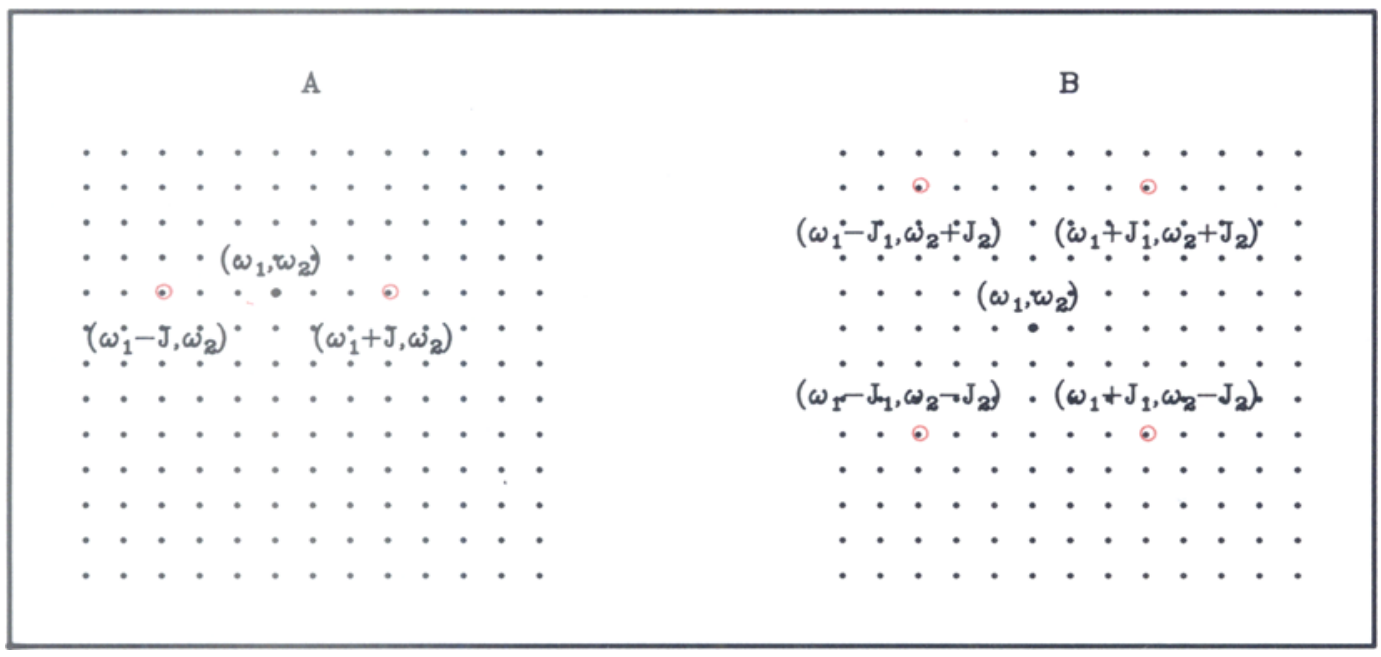

Figure 10. Points to which projection operators are applied in computing the response function for an arbitrary point using $A$ ) the symmetry filters of reference (1) and B) the pattern recognition procedure of references $(5,6$, 7).

operator is applied to a limited set of points about each possible center of symmetry. The symmetry filters described by BOLTON are one dimensional and selective for couplings with a particular value $\mathrm{J}_{\text {filter; }}$; the points used are those displaced $\pm J_{\text {filter }}$ in one dimension from the center point (Figure 10A). The template matching pattern recognition procedure of PFÄNDLER et al. is likewise selective for specific coupling constant values. It, however, considers coupling in both dimensions. The points utilized are those displaced $\pm J_{1}$ in one dimension and $\pm J_{2}$ in the other from the center point (Figure 10B). In contrast, the symmetry recognition procedure described here exploits the fact that all of the points comprising a spectral feature carry information about the location of the center of symmetry.

Another difference between the symmetry recognition procedure described here and symmetry filters or template matching procedures is that symmetry recognition is not $\mathrm{J}$ selective. A consequence is that the procedure is equally applicable to locating individual resonances or multiplets. The procedure also generalizes to cases in which the linewidths, coupling constants, or digital resolution in the two dimensions are different. In these cases the appropriate projection operators are those for the groups $\mathrm{D}_{2}$ and $C_{2 v}$, rather than $D_{2 d}$ and $C_{4 v}$, respectively. In addition, the procedure is applicable, in principle, to the out-of-phase component of phase sensitive spectra, providing a means of exploiting additional redundancy in the data.

\section{SUMMARY}

In summary, we have described a formal procedure for performing symmetry recognition on 2D-NMR data. The procedure utilizes group theory projection operators, and is consequently generalizable to the detection of features having arbitrary symmetry, whether individual resonances or multiplets. The concept of a projection operator in the image processing context serves to illustrate fundamental connections between template matching and symmetry filters and the present method, and provides a heuristic framework for exploring other aspects of invariance (or redundancy) in 2D-NMR spectra. In conjunction with simple significance tests, symmetry recognition provides a method suitable for practical application to large data sets. It should be emphasized that although symmetry recognition has been demonstrated here only in COSY spectra, the same methods 
can be applied to the dispersive quadrant of phase sensitive NOESY spectra. The practical application of symmetry recognition to both COSY and NOESY spectra of proteins will be reported elsewhere.

\section{ACKNOWLEDGEMENTS}

JCH gratefully acknowledges the hospitality of the Department of Chemistry of the Carlsberg Laboratory. We thank LesLey PEW, JetTe Holsøe, and Annette Pettersson for assistance in preparing the manuscript, PIA MIKKELSEN for technical assistance in preparation of the BSI 2, and Dr. MiChaEL BuRns for a critical reading of a draft of the manuscript. The Bruker AM 500 NMR spectrometer was provided by the Danish Natural Science Council and the Carlsberg Foundation. We thank the Institute of Organic Chemistry, the Technical University of Denmark for housing the spectrometer, and Dr. KLAus Bock for help and advice.

\section{REFERENCES}

1. Bolton, P.: Image analysis of proton two-dimensional chemical-shift-correlation spectra by application of symmetry rules. J. Magn. Reson. 70, 344 (1986)

2. Cotton, F. A.: Chemical applications of group theory, (Wiley-Interscience, eds.) New York (1971)

3. Hoch, J. C.: RIS Technical Memorandum 18t, Rowland Institute for Science, Cambridge, Mass., USA (1985)

4. JONASSEN, I.: Characteristics of hiproly barley I. Isolation and characterisation of two water-soluble high-lysine proteins. Carlsberg Res. Commun. 45 , 47-58 (1980)

5. Meier, B. U., G. Bodenhausen \& R. R. Ernst: Pattern recognition in 2D NMR. J. Magn. Reson. 60, 161 (1984)

6. Pfändler. P.\&G. Bodenhausen: Automated analysis of two-dimensional NMR spectra of mixtures by pattern recognition. J. Magn. Reson. 70, 71 (1986)

7. Pfändler.P., G. Bodenhausen, B. U. Meier \& R. R. ERNST: Toward automated assignment of nuclear magnetic resonance spectra: Pattern recognition in two-dimensional correlation spectra. Anal. Chem. 57, 2510-2516 (1985)

8. States, D. J., R. A. Haberkorn \& D. J. Ruben: A two-dimensional nuclear Overhauser experiment with pure absorption phase in four quadrants. J. Magn. Reson. 48, 286-291 (1982)

Accepted by S. O. Andersen and K. Schaumburg 